\title{
Effects of Didecyldimethylammonium Chloride on Sprague-Dawley Rats after Two Weeks of Inhalation Exposure
}

\author{
Cheol-Hong Lim and Yong-Hyun Chung \\ Toxicity Research Team, Occupational Safety and Health Research Institute, KOSHA, Daejeon, Korea
}

(Received May 15, 2014; Revised August 8, 2014; Accepted August 8, 2014)

\begin{abstract}
Didecyldimethylammonium chloride (DDAC) is used for various purposes, such as a fungicide for coolants, an antiseptic for wood, and disinfectant for cleaning. Despite the increasing likelihood of DDAC inhalation, available data on its toxicity from inhalation are scarce. Therefore, this study was aimed at confirming the toxicity of DDAC after inhalation exposure for $2 \mathrm{wk}$. Male Sprague-Dawley rats were exposed to approximately $0.15 \mathrm{mg} / \mathrm{m}^{3}, 0.6 \mathrm{mg} / \mathrm{m}^{3}$, and $3.6 \mathrm{mg} / \mathrm{m}^{3}$ DDAC aerosols in whole-body exposure chambers. After DDAC exposure for $2 \mathrm{wk}$, effects of DDAC on body weight, blood, bronchoalveolar lavage (BAL), and the lungs were verified. The mass median aerodynamic diameter of DDAC aerosols was $1.86 \mu \mathrm{m}$ and the geometric standard deviation was 2.75. The concentrations of DDAC aerosols for the low, medium, and high groups were $0.15 \pm 0.15 \mathrm{mg} / \mathrm{m}^{3}, 0.58 \pm 0.40 \mathrm{mg} / \mathrm{m}^{3}$, and $3.63 \pm 1.56 \mathrm{mg} / \mathrm{m}^{3}$, respectively. Body weight gain was significantly influenced by DDAC exposure. In the high group, a body weight decrease of $2.6 \mathrm{~g}$ was observed, whereas a $25.8 \mathrm{~g}$ increase was observed in the normal control group after the first 3 days. The low and medium groups showed $23.3 \mathrm{~g}$ and $20.4 \mathrm{~g}$ increases, respectively, after the first 3 days. Decreases in body weight were recovered during the next 4 days. In contrast, no changes were noted in hematological and blood biochemistry parameters after DDAC exposure. Furthermore, only mild effects were observed on bronchoalveolar cell differentiation counts and cell damage parameters in the BAL fluids of the medium and high groups. Although inflammatory cell infiltration and interstitial pneumonia were partially observed, fibrosis was not found in the lungs of the medium and high groups. In conclusion, body weight gain and the lungs were mainly affected by DDAC exposure. The noobserved-adverse-effect level (NOAEL) for DDAC was determined as $0.15 \mathrm{mg} / \mathrm{m}^{3}$.
\end{abstract}

Key words: Biocide, Didecyldimethylammonium chloride, Inhalation

\section{INTRODUCTION}

Chemical substances that are designed to kill organisms, especially microorganisms, are generally considered as biocides. Biocides are commonly used in medicine and agriculture. However, they also serve many other purposes. The European Union controls biocides by classifying them into 22 product types, with several comprising multiple subgroups: [1] Group 1 Disinfectants (type 1, human hygiene; type 2, disinfectants and algaecides not intended for direct application to humans or animals; type 3 , veterinary hygiene;

Correspondence to: Cheol-Hong Lim, Toxicity Research Team, Occupational Safety and Health Research Institute, KOSHA, 33930, Exporo, Yuseong-gu, Daejeon 305-380, Korea E-mail: limch@kosha.net

This is an Open-Access article distributed under the terms of the Creative Commons Attribution Non-Commercial License (http:// creativecommons.org/licenses/by-nc/3.0) which permits unrestricted non-commercial use, distribution, and reproduction in any medium, provided the original work is properly cited. type 4, food and feed area; type 5, drinking water), [2] Group 2 Preservatives (type 6, preservatives for products during storage; type 7 , film preservatives; type 8 , wood preservatives; type 9, fiber, leather, rubber, and polymerized materials preservatives; type 10 , construction material preservatives; type 11, preservatives for liquid-cooling and processing systems; type 12 , slimicides; type 13 , working or cutting fluid preservatives), [3] Group 3 Pest control (type 14 , rodenticides; type 15 , avicides; type 16 , molluscicides, vermicides, and products to control other invertebrates; type 17, piscicides; type 18, insecticides, acaricides, and products to control other arthropods; type 19, repellents and attractants; type 20, control of other vertebrates), and [4] Group 4 Other biocidal products (type 21, antifouling products; type 22, embalming and taxidermist fluids) (1). The United States Environmental Protection Agency classifies antimicrobial pesticides into 12 categories by their usage patterns: [1] agricultural premises and equipment; [2] food handling/storage establishments, premises, and equipment; [3] commercial, institutional, and industrial premises and 
equipment; [4] residential and public access premises; [5] medical premises and equipment; [6] human drinking water systems; [7] materials preservatives; [8] industrial processes and water systems; [9] antifouling coatings; [10] wood preservatives; [11] swimming pools; and [12] aquatic areas (2).

There is a high possibility of exposure to biocides through inhalation according to their usage pattern. However, the importance of inhalation toxicity for low vapor pressure and nonvolatile biocides, such as didecyldimethylammonium chloride (DDAC) has been underestimated until reports of the deaths of pregnant women and infants due to biocides added to humidifiers were reported. In Korea, numerous cases of interstitial lung disease in children every spring beginning in 2006 had been reported and it was suggested that humidifier disinfectants caused the lung disease $(3,4)$. Seventy-eight people, including 36 infants or toddlers, died in situations where additive biocides such as polyhexamethylene guanidine (PHMG), oligo(2-)ethoxy ethoxyethyl guanidine chloride (PGH), and 5-chloro-2-methyl-4-isothiazolin3-one (CMIT) were the cause (5).

DDAC is a typical quaternary ammonium biocide. It is used for indoor and outdoor hard surfaces, eating utensils, laundry, carpets, swimming pools, decorative ponds, re-circulating cooling water systems, etc. Inhalation exposure to DDAC is also estimated to be relatively low for various occupational handlers such as in agricultural premises and equipment, food handling/storage premises and equipment, and commercial, institutional and industrial premises and equipment. It is added directly to water to suppress microorganisms; the application rate of DDAC varies according to its usage, i.e., approximately 2 ppm for swimming pools, compared with 2,400 ppm for hospitals, health care facilities, and athletic/recreational facilities. DDAC is classified into acute oral toxicity category $3\left(\mathrm{LD}_{50}, 238 \mathrm{mg} / \mathrm{kg}\right)$ according to the Globally Harmonized System of Classification and Labeling of Chemicals (GHS). DDAC is also classified as highly irritating to the eye and skin. The no observed adverse effect level (NOAEL) for DDAC was suggested to be $10 \mathrm{mg} /$ $\mathrm{kg} /$ day according to the results of a chronic dietary toxicity study in dogs, or $45.5 \mathrm{mg} / \mathrm{kg} /$ day according to a sub-chronic oral feed toxicity study in Sprague Dawley (SD) rats. However, inhalation toxicity data for DDAC is rare, similar to other nonvolatile biocides. No repeated-inhalation toxicity studies have been reported, although the acute inhalation toxicity of DDAC is relatively high; DDAC is classified into category $2\left(\mathrm{LC}_{50}, 0.07 \mathrm{mg} / \mathrm{L}\right)$ according to the GHS $(6,7)$. Only Ohnuma et al. have instilled DDAC to mice and confirmed that $1,500 \mu \mathrm{g} / \mathrm{kg}$ of DDAC caused pulmonary inflammation and fibrosis (8). However, repeated-inhalation toxicity data is also necessary to conduct a more detailed risk assessment for workplace safety. Therefore, we exposed SD male rats to DDAC aerosols for $2 \mathrm{wk}$ and investigated its toxicity.

\section{MATERIALS AND METHODS}

Animals. Five-wk-old male specific pathogen-free SD rats were obtained from Central Lab Animal Inc. (Seoul, Korea). Rats were exposed to DDAC after a 2-wk acclimation period. During the experimental period, the rats were housed in a room with controlled temperature $\left(23 \pm 2{ }^{\circ} \mathrm{C}\right)$, humidity $(50 \pm 10 \%)$, and a 12-hr light/dark cycle. Rats were fed with filtered water and a rodent diet (LabDiet 5053, PMI Nutrition, St. Louis, MO, USA) ad libitum. General clinical symptoms, such as eye, skin, respiration, and pattern of movement were observed during the DDAC exposure period. This study was approved by animal ethics committee to ensure appropriate animal care for research.

\section{Generation of DDAC aerosol and exposure to SD male} rats. DDAC was purchased from Shin won Chemtrade Co. Ltd. (China). Aerosols of DDAC were generated using mist-generating equipment (VG-4R, Sibata Co. Ltd., Japan) in a whole-body exposure system (SIS-20RG, Sibata Co. Ltd., Japan). The system consisted of 4 chambers: one for the normal control group and 3 for the exposure groups. DDAC aerosols were generated by supplying air at a flow rate of 10 13 liters per min. The particle size distribution of DDAC was measured using a 9-stage Anderson sampler (Sibata Impactor 200913, Japan). Aerosols in the chamber were collected for $10 \mathrm{~min}$ at 28.3 liters per min around the respiratory site of experimental animals and the sample filter (800 mm, Pallflex, Japan) was measured in a microbalance (Kern 770, Germany). DDAC aerosols in the chamber were sampled using a XAD-2 resin tube $(8 \times 110 \mathrm{~mm}, 200 /$ $400 \mathrm{mg}$ XAD-2, SKC, PA, USA) and measured using ion chromatography (Dionex AS50, CA, USA). The analytical condition was $30 \mathrm{mM}$ sulfuric acid ( $>95 \%$, Aldrich, MO, USA) and acetonitrile (J.T. Baker, Pennsylvania, USA) (3: 7) with an IonPac AS7 column (9).

Bronchoalveolar lavage. Rats were anesthetized with isoflurane (Ilsung Pharmaceuticals Co., Seoul, Korea) and blood was collected from the abdominal aorta. Subsequently, the tracheae were cannulated and the lungs were lavaged 5 times with $3 \mathrm{ml}$ of calcium- and magnesium-free phosphate-buffered saline (PBS, $\mathrm{pH}$ 7.4). The lavaged fluids were centrifuged at 1,500 rpm for $10 \mathrm{~min}$ (Hanil Union $32 \mathrm{R}$, Incheon, Korea). The supernatants were stored at $-80^{\circ} \mathrm{C}$ for a later protein and lactate dehydrogenase (LDH) assay. The numbers of precipitated cells were counted with a Coulter Counter (Drew Science, Hemarvet 850, Miami Lakes, FL, USA) and the cells were then centrifuged with a Cyto centrifuge (Hanil Cellspin, Incheon, Korea). Centrifuged cells were stained in a Diff-Quick staining solution (Sysmex, Kobe, Japan) and differential counts of macrophages, lymphocytes, and polymorphonuclear leukocytes (PMNs) were performed by counting approximately 300 cells under a 
microscope at 100x magnification. The LDH and albumin in the lavage fluid were measured using a biochemistry analyzer (Toshiba TBA 20FR, Tokyo, Japan).

Hematology and blood biochemistry. Blood samples were collected from the abdominal aorta in blood-collecting tubes containing the anti-coagulant ethylenediaminetetraacetic acid (EDTA). Total white blood cells, neutrophiles, lymphocytes, monocytes, eosinophils, and basophils were counted using a veterinary multi-species hematology instrument (Drew Science, Hemavet 850, CT, USA). Total protein, blood urea nitrogen, creatinine, glucose, total bilirubin, total cholesterol, glutamic oxalacetic transaminase, gamma glutamyl transpeptidase, glutamic pyruvic transaminase, alkaline phosphatase, lactate dehydrogenase, and albumin were measured using a biochemical analyzer (Toshiba TBA-20FR, Japan).

Histopathology. The lungs were fixed in a $10 \%$ formalin solution containing neutral PBS and embedded in paraffin. After staining with hematoxylin and eosin, lung samples were examined by light microscopy at 400x magnification.

Statistical analysis. Results were presented as mean \pm standard deviation. Data were analyzed by analysis of variance (ANOVA) followed by post-hoc analysis based on the t-test or Mann-Whitney rank sum test to determine the differences between the compared groups. Statistical analyses were performed using SigmaPlot 12 (Systat Software Inc., San Jose, CA, USA). Differences were considered significant when the $p$-value was $<0.05$.

\section{RESULTS}

DDAC aerosols were generated appropriately according
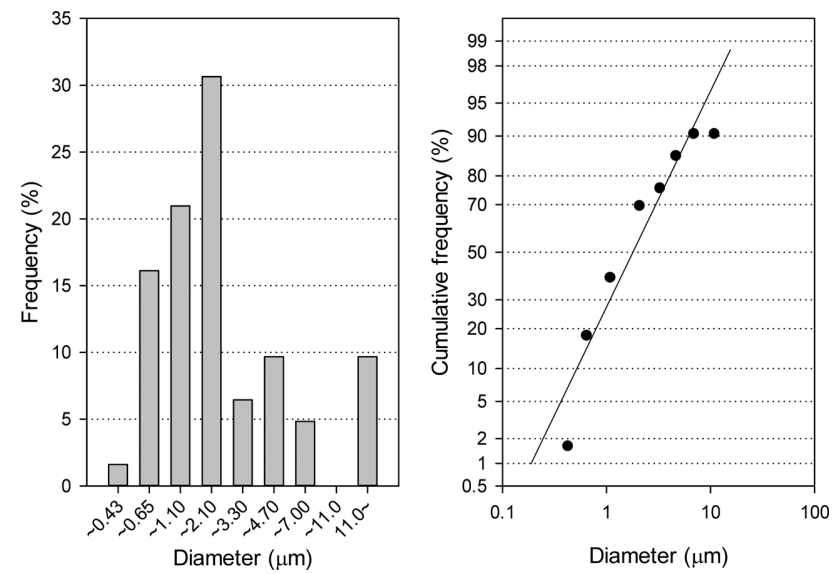

Fig. 1. Particle size distribution of didecyldimethylammonium chloride (DDAC) aerosols in a whole-body exposure inhalation chamber. to the OECD guides for inhalation toxicity. Namely, the mass median aerodynamic diameter was $1.86 \mu \mathrm{g} / \mathrm{ml}$ and the geometric standard deviation was 2.75 at $2.19 \mathrm{mg} / \mathrm{m}^{3}$ (Fig. 1). During the exposure period, the concentration of DDAC was adjusted to the target concentration; the concentrations for the low, medium, and high groups were $0.15 \pm 0.15$, $0.58 \pm 0.40,3.63 \pm 1.56$, respectively (Table 1 ).

Influence on body weight gain was the most prominent effect of DDAC on the rats. Body weight gain was significantly decreased by exposure to the high concentration (3.6 $\mathrm{mg} / \mathrm{m}^{3}$ ) of DDAC; a decrease of $2.6 \mathrm{~g}$ was observed in the high group after the first 3 days, whereas the normal control, low, and medium groups experienced $25.8 \mathrm{~g}, 23.3 \mathrm{~g}$, $20.4 \mathrm{~g}$ increases, respectively. Body weight gains were

Table 1. Concentrations of didecyldimethylammonium chloride (DDAC) aerosols in the whole-body inhalation chambers during a 2-wk exposure period

(unit, $\mathrm{mg} / \mathrm{m}^{3}$ )

\begin{tabular}{ccccc}
\hline \hline Method & Control & Low & Medium & High \\
\hline Concentrations & ND & $0.15 \pm 0.15$ & $0.58 \pm 0.40$ & $3.63 \pm 1.56$ \\
\hline
\end{tabular}

Values presented as mean \pm standard deviation.

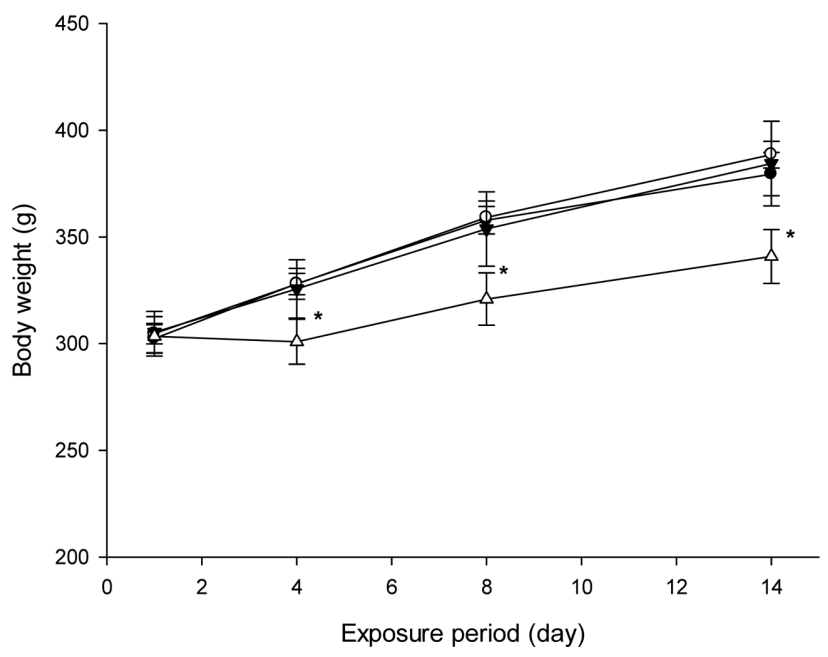

Fig. 2. Body weight changes of didecyldimethylammonium chloride (DDAC) exposed rats. ${ }^{*}, p<0.05$ vs control.

Table 2. Effects of didecyldimethylammonium chloride (DDAC) on blood hematology

\begin{tabular}{lcccc}
\hline \hline Parameter & Control & Low & Medium & High \\
\hline WBC & $4.92 \pm 0.50$ & $5.53 \pm 0.51$ & $6.05 \pm 0.92$ & $5.43 \pm 0.84$ \\
NE & $1.60 \pm 0.21$ & $1.68 \pm 0.30$ & $1.98 \pm 0.44$ & $1.93 \pm 0.42$ \\
LY & $1.50 \pm 0.48$ & $1.48 \pm 0.30$ & $1.73 \pm 0.46$ & $1.20 \pm 0.37$ \\
MO & $1.07 \pm 0.04$ & $1.36 \pm 0.30$ & $1.37 \pm 0.30$ & $1.14 \pm 0.29$ \\
EO & $0.74 \pm 0.54$ & $1.00 \pm 0.23$ & $0.96 \pm 0.38$ & $1.13 \pm 0.25$ \\
BA & $0.01 \pm 0.01$ & $0.02 \pm 0.02$ & $0.02 \pm 0.01$ & $0.03 \pm 0.02$
\end{tabular}

Values presented as mean \pm standard deviation.

WBC, White blood cell; NE, Neutrophil; LY, Lymphocyte; MO, Monocyte; EO, Eosinophil; BA, Basophil. 
Table 3. Effects of didecyldimethylammonium chloride (DDAC) on blood biochemistry

\begin{tabular}{|c|c|c|c|c|}
\hline Organ & Control & Low & Medium & High \\
\hline TP (mg/dl) & $5.85 \pm 2.47$ & $6.16 \pm 2.65$ & $6.12 \pm 2.60$ & $6.22 \pm 2.61$ \\
\hline $\operatorname{ALB}(\mathrm{mg} / \mathrm{dl})$ & $3.72 \pm 1.59$ & $3.92 \pm 1.68$ & $3.88 \pm 1.65$ & $3.92 \pm 1.65$ \\
\hline BUN (mg/dl) & $16.62 \pm 6.65$ & $17.21 \pm 7.09$ & $16.29 \pm 6.58$ & $15.10 \pm 5.96$ \\
\hline CRTN (mg/dl) & $0.63 \pm 0.25$ & $0.64 \pm 0.26$ & $0.62 \pm 0.26$ & $2.11 \pm 2.30$ \\
\hline T-BIL (mg/dl) & $0.09 \pm 0.04$ & $0.09 \pm 0.04$ & $0.09 \pm 0.04$ & $0.09 \pm 0.04$ \\
\hline ALT (IU/L) & $56.91 \pm 22.70$ & $73.37 \pm 29.56$ & $64.24 \pm 26.23$ & $80.46 \pm 32.24$ \\
\hline AST (IU/L) & $111.65 \pm 43.64$ & $160.54 \pm 62.97$ & $129.34 \pm 50.18$ & $156.78 \pm 66.46$ \\
\hline LDH (IU/L) & $2119.0 \pm 823.2$ & $2147.3 \pm 836.6$ & $2332.0 \pm 931.4$ & $2280.1 \pm 887.1$ \\
\hline ALP (IU/L) & $667.72 \pm 259.12$ & $662.59 \pm 260.43$ & $674.15 \pm 264.67$ & $576.45 \pm 224.78$ \\
\hline GLU (mg/L) & $122.02 \pm 49.25$ & $131.94 \pm 52.43$ & $126.22 \pm 49.12$ & $144.17 \pm 56.45$ \\
\hline T-CHO (mg/dl) & $46.87 \pm 19.16$ & $56.26 \pm 22.02$ & $58.35 \pm 22.71$ & $59.84 \pm 23.24$ \\
\hline
\end{tabular}

Values presented as mean \pm standard deviation $(n=5)$. TP, Total protein; ALB, albumin; BUN, blood urea nitrogen; CRTN, Creatinine; T-BIL, total bilirubin; ALT, alanine aminotransferase; AST, aspartate transaminase; LDH, lactate dehydrogenase; ALB, albumin; ALP, alkaline phosphatase; GLU, glucose; T-CHO, total cholesterol.

Table 4. Differential counts of bronchoalveolar lavage cells

\begin{tabular}{lcccc}
\hline \hline Cells & Control & Low & Medium & High \\
\hline Total cell & $1.272 \pm 1.135$ & $1.272 \pm 0.714$ & $2.152 \pm 1.195$ & $1.254 \pm 0.803$ \\
Macrophage & $1.196 \pm 1.058$ & $1.166 \pm 0.647$ & $1.884 \pm 1.104$ & $1.057 \pm 0.660$ \\
Lymphocyte & $0.069 \pm 0.077$ & $0.089 \pm 0.102$ & $0.158 \pm 0.235$ & $0.094 \pm 0.134$ \\
PMN & $0.008 \pm 0.004$ & $0.017 \pm 0.013$ & $0.110 \pm 0.079^{*}$ & $0.104 \pm 0.131$ \\
\hline
\end{tabular}

Values presented as mean $\pm \mathrm{SD}(\mathrm{n}=5) .{ }^{*}, p<0.05$ vs control.

recovered over the next 4 days in the low and medium groups. However, the body weight gain of the high group was not recovered until the end of the DDAC exposure period; the body weight gain of the high concentration exposure group was only $48.5 \%$ of that of normal control group (Fig. 2).

Compared to the body weight, there were no changes in hematological and blood biochemistry parameters (Table 2 and Table 3). Only mild effects were observed in the BAL cell differentiation counts and cytotoxicity parameters from BAL fluid. Namely, the PMNs of the medium and high group were mildly higher than those of the normal control group (Table 4), and the albumin concentration in the BAL fluid of the high concentration exposure group was significantly higher than that of the normal control group (Table 5).

The lung weights of the DDAC-exposed group were not changed compared to the normal control group (data not shown). Histopathological findings showed migration of

Table 5. Effects of didecyldimethylammonium chloride (DDAC) on cell damage indicator proteins in bronchoalveolar lavage fluid

\begin{tabular}{lrrcr}
\hline \hline & \multicolumn{1}{c}{ Control } & \multicolumn{1}{c}{ Low } & Medium & \multicolumn{1}{c}{ High } \\
\hline ALB (mg/dl) & $1.44 \pm 0.17$ & $1.62 \pm 0.15$ & $1.68 \pm 0.31$ & $1.94 \pm 0.33^{*}$ \\
LDH (IU/L) & $12.16 \pm 4.27$ & $10.27 \pm 5.37$ & $8.61 \pm 8.43$ & $13.84 \pm 10.27$ \\
\hline
\end{tabular}

Values presented as mean \pm standard deviation $(n=5) .{ }^{*}, p<0.05$ vs control. inflammatory cells, elimination of epithelial cells, and focal thickening of the alveolar wall caused by infiltration and proliferation in most of the lungs of DDAC-exposed rats. Furthermore, inflammatory cell infiltration and interstitial pneumonia were partially observed in the medium and high groups. However, fibrosis was not induced by DDAC exposure, although fibroblasts were found in some of the lungs of DDAC-exposed rats (Fig. 3).

\section{DISCUSSION}

DDAC is a broad-spectrum bactericidal and fungicidal biocide that is recommended for use in hospitals, hotels, and industry and it is frequently used in gynecology, surgery, ophthalmology, and pediatrics, and for the sterilization of surgical instruments and endoscopes, and for surface disinfection. Generally, the respiratory system is not exposed much to quaternary ammoniums like DDAC because their vapor pressure is relatively low. However, DDAC could be inhaled quite a lot if it is added to the water for humidifiers for the home. The background to this study was an accident where certain biocides were inhaled through the respiratory system and caused severe lung damage to pregnant women and infants in Korea (5). In Korea, many homes use humidifiers during the winter season. Most humidifiers for the home have adopted an ultrasonic method. The disadvantage of the ultrasonic method is that microorganisms in the water could be spread in vaporized water if humidifiers are 

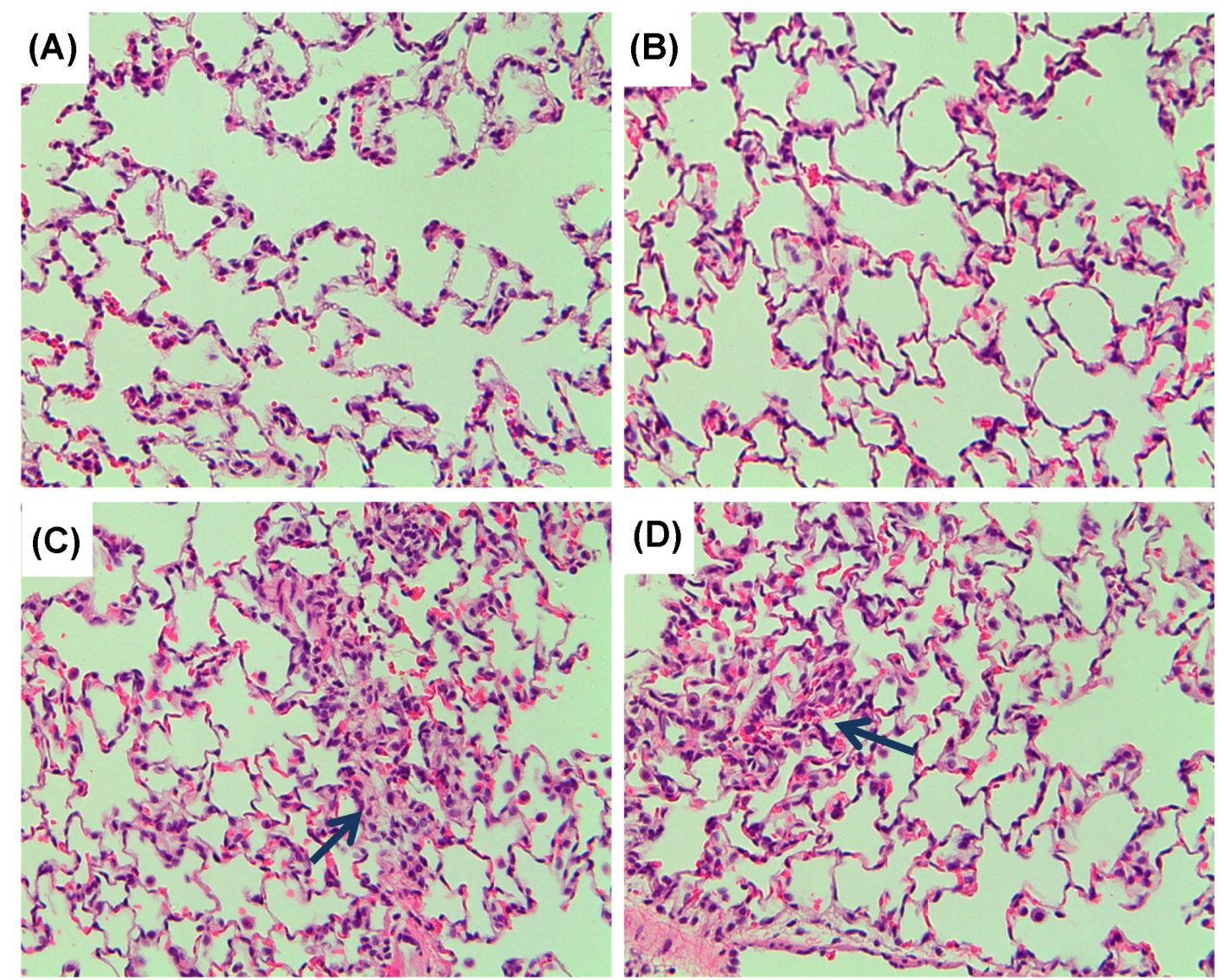

Fig. 3. Histopathology of the lungs exposed to didecyldimethylammonium chloride (DDAC). (A) control; (B) low group; (C) medium group; (D) high group. Arrows indicate migration of inflammatory cells, elimination of epithelial cells, and focal thickening of the alveolar wall by infiltration and proliferation.

not cleaned frequently. Unfortunately, attempts were made to use certain biocides to disinfect the water in humidifiers without proper hazard and risk assessment. Additionally, some biocidal products for humidifiers were regarded as harmless or nontoxic without proper inhalation toxicology data. The results were fatal; 78 people, including 36 infants or toddlers, died because of biocides. The main ingredients of the humidifier disinfectants were PHMG, PGH, and CMIT (5). These materials were selected as additives for humidifier disinfection because they have relatively low dermal toxicity compared to other biocides. As shown in the case of humidifier additives, biocides could be found everywhere around everyday necessities. However, there is little information on the inhalation toxicity of biocides. Therefore, the purpose of this study was to provide repeated-inhalation toxicity data, in order to provide a more detailed hazard and/or risk assessment.

In Korea, quaternary ammoniums, triazines, and isothiazols have been used as a preservative or disinfectant for cooling water. Among them, approximately 20 tons of DDAC, a quaternary ammonium, is used in the 17 workplaces annually in Korea (10) and more than 100 tons of it is used annu- ally in the European Union (11). Therefore, we selected DDAC as an experimental material to perform a repeatedinhalation toxicity study of biocides.

Weak inflammation was observed in rats instilled $150 \mu \mathrm{g} /$ $\mathrm{kg}$ of DDAC and severe congestive lung edema occurred in those instilled 1,500 $\mu \mathrm{g} / \mathrm{kg}$ of DDAC (8). A dose $1,500 \mu \mathrm{g} / \mathrm{kg}$, found by Ohmura to cause severe congestive lung edema in mice, was considered the highest concentration when determining concentrations for this repeated-inhalation toxicity study. The equivalent concentration for the instilled dose of $1,500 \mu \mathrm{g} / \mathrm{kg}$ was calculated to be inhaled $3.6 \mathrm{mg} / \mathrm{m}^{3}$; the respiratory volume per day for mice was considered 0.05 $\mathrm{m}^{3} /$ day and body weight was considered $0.03 \mathrm{~kg}$ to calculate the equivalent concentration. The medium and low concentrations were determined to be $0.6 \mathrm{mg} / \mathrm{m}^{3}$ and $0.15 \mathrm{mg} /$ $\mathrm{m}^{3}$, respectively.

The mass median aerodynamic diameter of the DDAC aerosol at $2.19 \mathrm{mg} / \mathrm{m}^{3}$ was $1.86 \mu \mathrm{m}$ and the geometric standard deviation was 2.75 . These values are within the proper range that the OECD recommends; the OECD recommended size of aerosols for inhalation toxicity is from 1 to $4 \mu \mathrm{m}$ and the geometric standard deviation from 1.5 to 3.0. 
The effects of DDAC on body weight changes were obvious whereas those on other measures were not clear. There were no significant differences in the blood hematological changes or blood biochemistry. Only mild effects were observed on the inflammatory responses in the BAL cell differentiation count and cell damage indicators, i.e., the number of PMNs in the medium and high groups were mildly higher than that in the normal control group, and the concentrations of albumin were significantly higher. Lung histopathological findings showed migration of inflammatory cells and thickening of alveolar walls in the medium and high groups. However, the severity of these was much milder compared to those in Ohnuma's report. Ohnuma observed fibrosis in the lungs of mice instilled with $1,500 \mu \mathrm{g} /$ $\mathrm{kg}$ of DDAC, the equivalent inhalation concentration to 3.6 $\mathrm{mg} / \mathrm{m}^{3}(8)$. It seems that the effects of DDAC on experimental animals are influenced by the exposure pattern because DDAC is exposed rapidly at the high concentration with a one-time instillation, whereas it is exposed slowly and repeatedly at the low concentration by inhalation. Increased effects on BAL cell differentiation, cytokines, and cell damage indicate that proteins recovered to a normal range by 13 days after $150 \mu \mathrm{g} / \mathrm{kg}$ of DDAC instillation in Ohnuma's experiment and these results may also explain the mild increase of PMNs and albumins in the BAL fluid in this study. It seemed that the effect on the body weight change was mainly due to decreased food consumption; in our other experiment, decreased food consumption was found in rats decreased body weight (data not shown).

In determining the exposure concentrations, we considered that no lethal effects were found at the highest concentration. Therefore, we determined the highest concentration to be $3.6 \mathrm{mg} / \mathrm{m}^{3}$. This concentration is 20 times lower than the acute $\mathrm{LC}_{50}$ in rats and we expected no lethal effect would be caused at this concentration. Body weights in the high group decreased $2.56 \mathrm{~g}$ during the first 3 days, whereas they were increased $25.8 \mathrm{~g}$ in the normal control group; we, therefore, considered the concentration for the high group was appropriate. In conclusion, the main targets were lung and body weight following 2-wk repeated-inhalation exposure to DDAC, and the NOAEL for DDAC should be considered as $0.15 \mathrm{mg} / \mathrm{m}^{3}$. We also suggest further study should be performed examining DDAC exposure by inhalation for longer because people could be exposed to many biocides over a sustained period.

\section{REFERENCES}

1. European Union (2012) Regulation (EU) No 528/2012 of the
European parliament and of the council of 22 May 2012 concerning the making available on the market and use of biocidal products, Annex V. Official Journal of the European Union, pp. 105-107.

2. United States Environmental Protection Agency (2011) Pesticide registration manual: Chapter 4 - Additional considerations for antimicrobial products. Updated August 2011. http:/ /www.epa.gov/opprd001/registrationmanual/ Assessed 11 April 2014.

3. Kim, K.W., Ahn, K., Yang, H.J., Lee, S., Park, J.D., Kim, W.K., Kim, J.T., Kim, H.H., Rha, Y.H., Park, Y.M., Sohn, M.H., Oh, J.W., Lee, H.R., Lim, D.H., Choung, J.T., Han, M.Y., Lee, E., Kim, H.Y., Seo, J.H., Kim, B.J., Cho, Y.A., Do, K.H., Kim, S.A., Jang, S.J., Lee, M.S., Kim, H.J., Kwon, G.Y., Park, J.H., Gwack, J., Youn, S.K., Kwon, J.W., Jun, B.Y., Pyun, B.Y. and Hong, S.J. (2014) Humidifier disinfectantassociated children's interstitial lung disease. Am. J. Respir. Crit. Care Med., 189, 48-56.

4. Yang, H.J., Kim, H.J., Yu, J., Lee, E., Jung, Y.H., Kim, H.Y., Seo, J.H., Kwon, G.Y., Park, J.H., Gwack, J., Youn, S.K., Kwon, J.W., Jun, B.Y., Kim, K.W., Ahn, K., Lee, S.Y., Park, J.D., Kwon, J.W., Kim, B.J., Lee, M.S., Do, K.H., Jang, S.J., Pyun, B.Y. and Hong, S.J. (2013) Inhalation toxicity of humidifier disinfectants as a risk factor of children's interstitial lung disease in Korea: a case-control study. PLoS One, $\mathbf{8}$, e64430.

5. The Korean Society of Environmental health and Toxicology (2011) Environmental and Toxicology Forum Report on Humidifier Biocide, Seoul, Korea.

6. United States Environmental Protection Agency (2006) Reregistration eligibility decision for aliphatic alkyl quaternaries (DDAC), EPA739-R-06-008. United States Environmental Protection Agency, Washington, D.C., pp. 1-37.

7. United State Environmental Protection Agency (2006) Draft Didecyl Dimethly Ammonium Chloride (DDAC) Risk Assessment, Office of Pesticide Programs Antimicrobials Division, V.A., pp. 9-32.

8. Ohnuma, A., Yoshida, T., Tajima, H., Fukuyama, T., Hayashi, K., Yamaguchi, S., Ohtsuka, R., Sasaki, J., Fukumori, J., Tomita, M., Kojima, S., Takahashi, N., Takeuchi, Y., Kuwahara, M., Takeda, M., Kosaka, T., Nakashima, N. and Harada, T. (2010) Didecylmethylammonium chloride induces pulmonary inflammation and fibrosis in mice. Exp. Toxicol. Pathol., 62, 643-651.

9. Yang, J.S., Choi, S.B., Park, S.Y. and Lee, S.B. (2012) Analysis of didecyldimethylammonium chloride (DDAC) aerosol in inhalation chamber. Anal. Sci. Technol., 25, 307-312.

10. Ministry of Environment (2007) $3^{\text {rd }}$ Report on circulating chemical list, Korea Ministry of Environmental Protection, Seoul, Korea.

11. European Chemical Agency (2014) Registered substances, http://echa.europa.eu/web/guest/information-on-chemicals/ registered-substances Assessed 11 April 2014. 